\title{
Mitos sobre la prevención y el control de las enfermedades no transmisibles en América Latina
}

María Cristina Escobar, D r. en M., ${ }^{(1)}$ Andrés Petrásovits, Ph. D., M.P.H., ${ }^{(2)}$

Armando Peruga, Dr. en M., Dr. en S.P. ${ }^{(3)} N$ yvea Silva, Ph. D, ${ }^{(4)}$

MarcelaVives, D r. en Microbiol y Q uím. C lín. ${ }^{(5)}$ Sylvia Robles, M. en C., D r. en M. ${ }^{(6)}$

\begin{abstract}
Escobar MC, Petrásovits A, Peruga A, Silva N, Vives M, Robles S. Mitos sobre la prevención y el control de las enfermedades no transmisibles en América Latina Salud Publica Mex 2000;42:56-64.
\end{abstract}

\section{Resumen}

El incremento de las enfermedades no transmisibles (ENT) y la posibilidad de evitarlo hacen apremiante la puesta en marcha o el reforzamiento de los programas preventivos de este tipo de padecimientos en América Latina. Sin embargo, existe una serie de mitos alrededor de las EN T, que dificulta la aplicación de dichos programas; en estos mitos subyace la idea de que éstas son: a) degenerativas e incurables; b) enfermedades de la vejez, y c) enfermedades de los ricos. A simismo, existen mitos según los cuales los programas preventivos de las EN T son: a) difíciles de implementar; b) costosos, y c) ineficaces. En este trabajo se presentan datos que demuestran que tales mitos no son ciertos, y se discuten los retos a vencer para encontrar una política equilibrada de salud que resalte la importancia de las ENT sin olvidar la de las otras enfermedades.

Palabras clave: enfermedades no transmisibles / prevención y control;América Latina

\author{
Escobar MC, Petrásovits A, Peruga A, \\ Silva N, Vives M, Robles S. \\ Myths on prevention and control \\ of non-communicable diseases \\ in Latin A merica. \\ Salud Publica Mex 2000;42:56-64.
}

\section{A bstract}

Given the increase of the burden of non-communicable diseases (NCD) and the possibility to avo id it, it is urgent to implement or strengthen NCD preventive programs in Latin America. However, many myths hinder the implementation of NCD programs. Myths on NCD include:a) NCD are degenerative and incurable; $b$ ) they are diseases of the elderly;c) they are diseases of the rich. Like wise there are myths about NCD preventive programs are: a) difficult to implement, b) expensive, and c) ineffective.W e present data that demonstrate how these myths are untrue and discuss the challenges to find a balanced health policy that emphasizes the importance of NCD without overlooking other diseases.

Key words: non-communicable diseases/prevention and control; Latin America

(1) Presidenta del Comité de Gerencia de la Red Panamericana de Programas CARMEN (Conjunto de Acciones para la Reducción Multifactorial de Enfermedades No Transmisibles) y directora ejecutiva del Programa CARMEN en Chile.

(2) Coordinador federal de la Iniciativa de Salud del Corazón de Canadá (CARMEN -Canadá).

(3) Asesor regional del Programa de Enfermedades no Transmisibles de la O rganización Panamericana de la Salud (O PS).

(4) Directora de CARMEN en Puerto Rico.

(5) Directora de CARMEN en Costa Rica.

(6) Coordinadora del Programa de Enfermedades no Transmisibles de la O PS. 
E n casi todos los países de América Latina y el Caribe, las transformaciones sociales, económicas, demográficas y epidemiológicas de las últimas décadas han contribuido a la aparición de nuevas prioridades de salud. Entre estas últimas destacan, por su importante aumento, las enfermedades no transmisibles (ENT), que incluyen problemas cardiovasculares, cánceres, diabetes y padecimientos renales relacionados con la hipertensión, entre otros. ${ }^{*}$ En 1985, por cada 10 muertes debidas a una enfermedad transmisible, se producían 15 por una ENT, y hoy se producen 34. Son varias las causas que explican este cambio, todas ellas interrelacionadas: se ha producido una rápida urbanización e industrialización; la fecundidad ha bajado y la esperanza de vida ha subido, con el consiguiente envejecimiento de la población; ha habido cambios en los estilos de vida y, finalmente, el acceso a los servicios de salud y su efectividad han mejorado. Como resultado de todo ello, las ENT son ahora las principales causas de muerte y lo seguirán siendo en el futuro. Se prevé que para el año 2015, por cada 10 defunciones atribuidas a una causa infecciosa, habrá 70 fallecimientos por causas no transmisibles. ${ }^{1}$

Las ENT afectan a todos los grupos socioeconómicos de la sociedad e imponen dos tipos de cargas: por una parte, afectan la productividad de los individuos y su capacidad de generar ingresos y, por la otra, originan un mayor consumo de servicios sociales y de salud, generalmente de alto costo. De hecho, el progresivo envejecimiento de la población y el constante incremento de las ENT provocará un crecimiento significativo de la demanda de los servicios curativos.

Las ENT más frecuentes son evitables en gran medida. La prevención está dirigida a evitar la aparición de los factores de riesgo que anteceden a la presentación de la enfermedad, o bien, a tratar dichos factores lo antes posible una vez que han aparecido. El incremento de las ENT y la posibilidad de evitarlo hacen apremiante e impostergable la puesta en marcha o el reforzamiento de los programas preventivos latinoamericanos y caribeños, destinados a modificar la prevalencia de los factores de riesgo, particularmente entre los adolescentes y adultos jóvenes. Sin embargo, existe una serie de preconcepciones o mitos ${ }^{2}$ relativos a las enfermedades crónicas, que dificulta o impide la puesta en marcha de dichos programas y de la cooperación técnica que los acompaña.

\footnotetext{
* A los efectos del análisis presentado en este artículo, las enfermedades no transmisibles corresponden a los siguientes códigos de la Novena Clasificación Internacional de Enfermedades: 140-242, 244-259, 286-319, 323-380, 383-459, 467-479, 488-613, 617-629, 680-759. Están expresamente excluidas las lesiones externas.
}

\section{Mito 1. Las enfermedades no transmisibles son degenerativas e incurables}

Existe la idea de que la aparición de las ENT es consecuencia de un proceso biológico degenerativo inexorable que acompaña al envejecimiento, que dicho proceso es generalmente irreversible y que, por tanto, no es mucho lo que se puede hacer desde el punto de vista de las intervenciones de prevención o tratamiento. Sin embargo, la evidencia científica disponible muestra que la probabilidad de padecer este tipo de enfermedad disminuye sustancialmente cuando se elimina o modera la exposición a sus factores de riesgo, y que hoy existen tratamientos efectivos que disminuyen sus complicaciones y su letalidad. A modo ilustrativo, se muestran algunos ejemplos de lo indicado por esta evidencia.

Tradicionalmente se ha considerado que padecer una ENT, especialmente el cáncer, supone una sentencia de muerte que tarde o temprano se cumple. Afortunadamente esto no es así, ya que se conocen nuevos tratamientos que reducen cada vez más significativamente la letalidad de algunas. Un claro ejemplo lo constituyen los tumores malignos de testículo, así como algunos tipos de leucemia y linfomas, particularmente en niños, cuya sobrevida es muy prolongada y cuya remisión es muchas veces total después de la aplicación de quimioterapia, de tal manera que se pueden considerar curados. Otros tumores malignos, como los de cuello y cuerpo del útero, mama, colon, próstata, cavidad oral y el propio melanoma, se pueden curar mediante cirugía con o sin radioterapia y quimioterapia, si se detectan oportunamente. Sin embargo, hay que advertir que la sobrevida de los pacientes que padecen alguno de estos tumores, depende en gran medida de la accesibilidad y la disponibilidad del tratamiento, que varía en los diferentes contextos. ${ }^{3}$

También hay ejemplos de la capacidad para prevenir las complicaciones de las ENT. Por ejemplo, dos estudios han demostrado que el control intensivo de la glicemia, tanto con insulina como con hipoglicemiantes, reduce significativamente las complicaciones microvasculares de ambos tipos de diabetes mellitus. ${ }^{4,5}$

En cada uno de estos estudios, el riesgo de retinopatía se redujo en 63 y $21 \%$, respectivamente, y se observó una reducción importante de la prevalencia de albuminuria (54 y $34 \%$, respectivamente), que es un marcador de la nefropatía. Ello demuestra que las complicaciones, consideradas antes como parte inevitable de la historia natural de la enfermedad, en este momento se pueden evitar significativamente, y que es posi- 
ble mejorar la calidad de vida de las personas que viven con diabetes, si se brinda el tratamiento adecuado.

La literatura científica ha presentado en numerosas ocasiones pruebas de los efectos beneficiosos sobre la salud que produce intervenir en los factores de riesgo de las ENT. Dejar de fumar es un claro ejemplo. Desde hace tiempo se sabe que, entre fumadores, la incidencia acumulada de reinfartos tras un primer infarto no mortal de miocardio, es 50\% menor entre aquellos que abandonaron el hábito tabáquico tras el primer infarto, respecto de quienes siguieron fumando. ${ }^{6}$ También se sabe que la tasa de mortalidad por cáncer de pulmón disminuye sustancialmente a medida que aumenta la duración de la abstinencia tabáquica en la población, aproximándose mucho a la tasa de mortalidad entre los no fumadores. La tasa de mortalidad en una población de ex fumadores, que no han fumado durante más de 20 años, es aproximadamente cuatro veces menor que entre los fumadores. ${ }^{7}$ Incluso, la capacidad pulmonar se deteriora menos rápidamente entre los que dejaron de fumar que entre los fumadores, y este deterioro decrece en la medida en que disminuye el periodo en el que se tuvo el hábito tabáquico. ${ }^{8}$

La supresión de la influencia de determinados factores de riesgo no sólo puede moderar o detener la evolución de una enfermedad no transmisible, sino que, bajo determinadas circunstancias, puede revertir su historia natural. Tal es el caso de la ateroesclerosis, ${ }^{9}$ el principal motivo de enfermedad cardiovascular, así como la causa más importante de muerte y discapacidad en América Latina y el Caribe. ${ }^{10}$ Lo que recientemente sólo se había observado en animales, se ha podido probar en seres humanos. La placa ateromatosa puede retroceder a estadios anteriores de su evolución, si se reduce sustancialmente la concentración de lípidos en el torrente sanguíneo mediante cambios dietéticos, el incremento de la actividad física, la medicación hipolipemiante, o bien, mediante la combinación de estas intervenciones. Resultan esperanzadores aquellos estudios ${ }^{11,12}$ que destacan la importancia de las intervenciones de promoción de la salud; uno de ellos, por ejemplo, indica que la evolución de la placa ateromatosa se detiene cuando el nivel de actividad física alcanza un promedio de $1500 \mathrm{kcal}$ por semana, y que retrocede cuando rebasa las 2200 kcal semanales. ${ }^{11}$ Asimismo, se ha visto que el retroceso de la placa reduce la incidencia de problemas coronarios. ${ }^{13}$

Por tanto, la evidencia científica sugiere que la historia natural de las ENT no está determinada por azares incontrolables o por un deterioro biológico inexorable producido por el envejecimiento, sino por fac- tores ambientales modificables. En consecuencia, no hay razones para creer ni en la fatalidad de la evolución de las ENT ni en la incurabilidad de todas ellas.

\section{Mito 2. Las enfermedades no transmisibles son padecimientos de la vejez}

Los adultos jóvenes (15-59 años de edad) comparten con los adultos mayores (60 y más) el "privilegio" de morir y enfermar más de ENT que de otras causas. Es cierto que la mayor proporción de casos y defunciones por ENT se concentra en adultos mayores; sin embargo, en el grupo de adultos jóvenes la probabilidad de morir por una causa no transmisible es mayor que la de fallecer por causas transmisibles y maternas combinadas, en todas y cada una de las regiones del mundo. ${ }^{14}$ De la lectura del cuadro I se puede concluir que en el mundo menos desarrollado los mayores de 15 años mueren más frecuentemente de una causa no transmisible que de causas infecciosas y maternas combinadas. Esto también es cierto para los países más desarrollados; sin embargo, en estas naciones, si la mortalidad por causas no transmisibles es más baja que en los países en desarrollo, la mortalidad por causas infecciosas y materno-infantiles es todavía mucho menor $y$, por consiguiente, la diferencia entre mortalidad por enfermedades no transmisibles y aquella debida a padecimientos transmisibles es mayor que en los países menos desarrollados. Así, en el grupo de edad donde las defunciones se consideran prematuras (15 a 59 años), el conjunto dominante de causas de muerte es el de las no transmisibles, tanto en América Latina como en el resto de los países menos desarrollados.

\section{Cuadro I \\ Razón ENTRE LA TASA DE MORTALIDAd POR CAUSAS NO TRANSMISIBLES Y LA TASA DE MORTALIDAD POR CAUSAS INFECCIOSAS Y MATERNO-INFANTILES EN LOS PAÍSES MENOS DESARROLLADOS, POR GRUPO DE EDAD. Estimación PARA El año 2000}

Región del mundo \begin{tabular}{c} 
Grupo de edad \\
\hline $0-14 \quad 15-59 \quad 60$ y más
\end{tabular}

\begin{tabular}{lllr} 
Países menos desarrollados & 0.2 & 2.5 & 8.5 \\
\hline América Latina y el C aribe & 0.2 & 2.6 & 10.0 \\
\hline Resto de países menos desarrollados & 0.2 & 2.5 & 8.3
\end{tabular}

N ota: la razón fue calculada con los datos de la referencia 14

salud pública de méxico / vol.42, no.1, enero-febrero de 2000 
En América Latina y el Caribe, $81 \%$ de los años de vida saludables perdidos por la combinación de la mortalidad y la discapacidad ocasionada por las ENT ocurre antes de los 60 años de edad. La carga que supone las ENT en las edades más jóvenes queda igualmente ilustrada por la prevalencia creciente de algunos factores de riesgo de estas enfermedades como, por ejemplo, la prevalencia del sobrepeso en niños menores de cinco años. Este problema, definido como el porcentaje de niños con un peso superior en una desviación estándar a la media de la distribución, ha hecho su aparición en América Latina y el Caribe, cuando 20 años atrás era prácticamente desconocido. De los 13 países latinoamericanos para los que existen datos al respecto, en tres la prevalencia oscila entre 5 y $9 \%$; en nueve de ellos varía entre 10 y 19\%, y en uno (Perú) alcanza $22 \%{ }^{15}$

\section{Mito 3. Las enfermedades no transmisibles son padecimientos de los ricos}

A veces se descarta la necesidad de actuar contra las ENT o de proveer cooperación técnica con el argumento de que éstas son un problema exclusivo de los países ricos. La realidad es que en los países pobres o menos desarrollados no sólo se produce la mayor parte de las defunciones por ENT en números absolutos, sino que además la tasa de mortalidad por causas no transmisibles es más alta que en las naciones más industrializadas, tanto en hombres y mujeres como entre los más jóvenes y los menos jóvenes. En el cuadro II se presentan las tasas de mortalidad por las causas referidas, notificadas en las diversas regiones del mundo; se puede observar que las cifras para América Latina y el Caribe, comparadas con las de los países desarro-

\section{Cuadro II \\ TASAS DE MORTALIDAD POR CAUSAS NO TRANSMISIBLES, POR REGIONES DEL MUNDO Y GRUPO DE EDAD. Estimación PARA EL AÑo 2000}

\begin{tabular}{lccc} 
Región del mundo & \multicolumn{3}{c}{ Grupo de edad } \\
\cline { 2 - 4 } & $0-14$ & $15-59$ & 60 y más \\
Países desarrollados & 27.1 & 175.5 & 3539.6 \\
\hline Países menos desarrollados & 91.7 & 221.9 & 4426.0 \\
\hline América Latina y el Caribe & 65.4 & 187.7 & 3497.3 \\
\hline Resto de los países menos desarrollados & 94.6 & 225.8 & 4526.6
\end{tabular}

N ota:Tasas por 100000 habitantes calculadas con los datos de la referencia 14

salud pública de méxico / vol.42, no.1, enero-febrero de 2000 llados, son notablemente más altas entre los niños, ligeramente más elevadas en los adolescentes y adultos jóvenes, y muy similares entre los adultos mayores. Estos datos ponen en entredicho la creencia de que las no transmisibles son enfermedades de los países ricos.

Además, también existen datos que muestran que dentro de cada país la morbilidad y la mortalidad por ENT son más altas entre los grupos más desfavorecidos de la sociedad. En Brasil, por ejemplo, existen datos que indican que la mortalidad general es entre $3 \mathrm{y}$ 4 veces mayor en los trabajadores de categorías laborales más bajas que en los de las más altas. ${ }^{16}$ Datos más recientes de los mismos autores muestran que este gradiente se reproduce para la mortalidad por enfermedades cardiovasculares. Concretamente, el riesgo de morir por estas enfermedades es tres veces mayor en las categorías ocupacionales inferiores que en las superiores y siete veces mayor cuando se trata de la enfermedad congestiva del corazón.*

Otro ejemplo es el cáncer del cuello del útero. Este problema es más frecuente entre las mujeres de menor nivel educativo, según lo demuestran datos de Quito, Ecuador. ${ }^{17}$ Además, estos datos indican que $52 \%$ de las mujeres sin ninguna instrucción, en comparación con $22 \%$ de las mujeres con más nivel educativo, presentan tumores malignos avanzados (estadios III y IV) y de peor pronóstico, cuando son diagnosticadas por primera vez.

En un estudio realizado en cinco ciudades de América Latina (Bogotá, Colombia; Río de Janeiro y Sao Paulo, Brasil; Santiago de Chile y Temuco, Chile) se investigó la relación entre el nivel educativo y la prevalencia de varios factores de riesgo de las ENT. ${ }^{18}$ En algunas de las ciudades no se observó una asociación, pero en otras se vio que, a mayor nivel educativo, menor era el índice de masa corporal y la presión arterial sistólica. En Porto Alegre, Brasil, y Valparaíso, Chile (cuadro III), sendos estudios también presentan una relación inversa entre el nivel socioeconómico y la prevalencia de la mayoría de los factores de riesgo investigados. ${ }^{19,20}$

En resumen, se sabe que el riesgo de padecer un problema no transmisible es mayor en los países con menos recursos y que, en la población de cada país, hay diferencias sociales respecto de la frecuencia con la que ocurren las ENT. La distribución social de estas enfermedades y sus factores de riesgo está mediada por factores complejos, cuya naturaleza y dinámica

\footnotetext{
* Duncan BB, Osvaldt AB, Serrate-Mengue S, Rumel D. Cardiovascular mortality is highest among less skilled workers in Sao Paulo, Brazil. Comunicación personal.
} 


\section{Cuadro III \\ Prevalencia de factores de Riesgo de enfermedades CARDIOVASCULARES EN 3120 ADULTOS DE 25 A 64 años, según estrato socioeconómico. Chile, 1996}

\begin{tabular}{lrrrr} 
& \multicolumn{5}{c}{ Estrato socioeconómico } \\
\cline { 2 - 5 } Factor de riesgo & $\begin{array}{c}\text { Alto } \\
(\%)\end{array}$ & $\begin{array}{c}\text { Medio } \\
(\%)\end{array}$ & $\begin{array}{c}\text { Bajo } \\
(\%)\end{array}$ & p* \\
Colesterol HDL $\leq 35 \mathrm{mg} \%$ & 13.8 & 16.1 & 12.6 & 0.20 \\
\hline Colesterol total $\geq 200 \mathrm{mg} \%$ & 49.0 & 47.1 & 45.7 & 0.68 \\
\hline Hipertensión arterial $\geq 140 / 90 \mathrm{~mm} \mathrm{Hg}$ & 9.3 & 9.8 & 14.2 & 0.00 \\
\hline Sedentarismo & 78.9 & 83.1 & 89.4 & 0.00 \\
\hline Sobrepeso IMC $\geq 25$ & 56.4 & 61.0 & 65.3 & 0.00 \\
\hline Tabaquismo (fumadores actuales) & 39.8 & 39.5 & 42.9 & 0.22 \\
*p N o hay una diferencia significativa entre la prevalencia de los factores \\
de riesgo por estrato socioeconómico
\end{tabular}

Fuente: Programa CARMEN . Encuesta de base. Chile: Ministerio de Salud/ O rganización Panamericana de la Salud, 1997

todavía no es entendida completamente, ${ }^{21}$ pero existe suficiente evidencia para afirmar que en América Latina y el Caribe los grupos más desfavorecidos son los que soportan la mayor parte de la carga de las ENT.

\section{Mito 4. Los programas son difíciles de implementar}

El componente de cambio de comportamiento que tienen muchas de las intervenciones de prevención de las ENT, ha hecho presumir que dichas intervenciones tienen una dificultad inherente en su aplicación, porque cambiar el comportamiento supuestamente es más difícil que aplicar un componente de servicio como los programas de vacunación, o el control de vectores, o la creación de infraestructuras para el saneamiento del agua. Frente a esto hay que hacer dos consideraciones.

La primera es que, en la práctica, programas que aparentemente son más sencillos en el plano de la concepción técnica (generalmente basados en el modelo biomédico), también necesitan de un largo periodo para obtener los resultados esperados. El éxito de programas como la erradicación de la viruela, todavía no se ha repetido con otras enfermedades infecciosas prevenibles por vacunación desde hace más de dos décadas, aunque para el presente año está prevista la erradicación de la poliomielitis -lograda ya en las Américas- en una escala mundial. Otras enfermedades infecciosas pueden ser eliminadas o erradicadas antes del año 2010,22 sin embargo, en muchos países otros "viejos" padecimientos infecciosos, como el paludismo y la tuberculosis, todavía no tienen un horizonte temporal para su control. ${ }^{23}$

La segunda consideración es que las intervenciones preventivas que típicamente han recibido mayor atención de la cooperación técnica, como las de las enfermedades transmisibles, las relativas a las condiciones materno-infantiles o las relacionadas con los problemas de desnutrición, tienen también un componente sustancial de cambio de comportamiento, lo que invalida la presuposición de que las intervenciones en las ENT son inherentemente más difíciles. Tal es el caso de las medidas higiénicas necesarias para evitar infecciones transmitidas por medio del agua o los alimentos, o el cambio de comportamiento que exige el uso adecuado de los servicios de control prenatal o los de planificación familiar.

No se trata de desacreditar los logros obtenidos en la prevención y el control de otras enfermedades, que son muchos. Más bien se trata de reconocer las dificultades inherentes a cualquier programa de salud. Ciertamente existen obstáculos para la ejecución de los programas dirigidos a prevenir las ENT o a promocionar comportamientos saludables, pero no son necesariamente ni mayores ni menores que los de otras áreas, sino simplemente específicos de este tipo de problemas. $^{24}$

\section{Mito 5. La prevención de las enfermedades no transmisibles es cara}

Existe la creencia de que en los países menos desarrollados el costo de la prevención de las ENT es mayor que el de la de otros problemas; es decir, que las intervenciones para prevenir las ENT son menos costoefectivas que aquellas relacionadas con problemas a los que la cooperación técnica ha prestado tradicionalmente más atención. Una revisión de las evidencias existentes al respecto indica que esto no es necesariamente cierto. ${ }^{25}$ En el cuadro IV se puede ver que, por ejemplo, algunas intervenciones antitabáquicas son igualmente costo-efectivas que algunos programas de vacunación como el de la polio (triple vírica) o inclusive más que el del cólera.

Los programas de detección oportuna de cáncer de cuello de útero o de mama, o los programas integrados de prevención primaria y secundaria de las enfermedades cardiovasculares, son más costo-efectivos que algunas intervenciones preventivas de las diarreas (programas para mejorar prácticas higiénicas personales y domésticas) o que algunos programas de control de vectores de la malaria o el dengue. En cualquier caso, hay que considerar que las intervencio- 


\begin{tabular}{|c|c|c|c|c|}
\hline Costo & $\begin{array}{l}\text { Cuadr } \\
\text { EFECTIVIDAD DE LAS INTERVENCIONES DE SALUD } \\
\text { Estrategia de salud pública }\end{array}$ & $\begin{array}{l}\text { O IV } \\
\text { PÚBLIC }\end{array}$ & $\begin{array}{l}\text { CLÍNICAS PARA CADA TIPO DE PROBLEI } \\
\text { Estrategia clínica }\end{array}$ & EMA \\
\hline Problema & Intervención & $\begin{array}{l}\text { Costo por } \\
\text { AVISA }\end{array}$ & Intervención & $\begin{array}{l}\text { osto por AVISA } \\
\text { en dólares }\end{array}$ \\
\hline Diarreas & $\begin{array}{l}\text { Vacuna contra el rotavirus ( } 80 \% \text { efectividad) } \\
\text { Vacuna contra el cólera ( } 70 \% \text { efectividad) } \\
\text { Vacuna contra el sarampión ( } 85 \% \text { efectividad) } \\
\text { Cambio de comportamiento para mejorar la higiene } \\
\text { personal y doméstica } \\
\text { Promoción del amamantamiento materno } \\
\text { Educación para el destete }\end{array}$ & $\begin{array}{r}10 \\
75 \\
10 \\
\\
170 \\
30 \\
30\end{array}$ & Educación y terapia de rehidratación oral & $35-350$ \\
\hline Paludismo & $\begin{array}{l}\text { Control de vectores (según incidencia, tipo de mosquito } \\
\text { y área geográfica) }\end{array}$ & $5-520$ & $\begin{array}{l}\text { Tratamiento de casos detectados pasiva- } \\
\text { mente en zonas de endemicidad moderada } \\
\text { a alta y con control de vectores }\end{array}$ & $200-500$ \\
\hline IRA & $\begin{array}{l}\text { Tamizaje y derivación según mortalidad } \\
\text { Promoción de amamantamiento materno } \\
\text { Suplementación calórico-proteica } \\
\text { Vacuna pneumocócica (18 meses) }\end{array}$ & $\begin{array}{r}20-50 \\
50 \\
65 \\
70\end{array}$ & Tratamiento antibiótico de neumonía en niños & os $20-50$ \\
\hline ETS & $\begin{array}{l}\text { Educación para reducir número de parejas e incrementar } \\
\text { el uso del condón }\end{array}$ & $1-50$ & Tratamiento antibiótico de ETS & $1-55$ \\
\hline Poliomielitis & Vacuna triple (según incidencia y mortalidad) & $20-40$ & & \\
\hline Sarampión & $\begin{array}{l}\text { Vacuna (según incidencia, edad de vacunación, } \\
\text { dosis y antígeno) }\end{array}$ & $2-30$ & & \\
\hline Tuberculosis & BCG administrada con la triple vírica & 7 & Q uimioterapia con hospitalización & 3 \\
\hline Diabetes & $\begin{array}{l}\text { Prevención primaria mediante educación de DN ID } \\
\text { Tamizaje de intolerancia a la glucosa y educación }\end{array}$ & $\begin{array}{l}\text { No se sabe } \\
\text { No se sabe }\end{array}$ & $\begin{array}{l}\text { Control metabólico con hipoglicemiantes } \\
\text { orales y educación de DN ID } \\
\text { Inyección de insulina y educación }\end{array}$ & $\begin{array}{r}25 \\
240\end{array}$ \\
\hline Cáncer & $\begin{array}{l}\text { Detección oportuna del cáncer cervicouterino } \\
\text { (prueba de Papanicolaou) } \\
\text { Examen clínico anual de mama } \\
\text { Educación para abandonar el consumo de tabaco }\end{array}$ & $\begin{array}{r}100 \\
50 \\
20\end{array}$ & $\begin{array}{l}\text { Cirugía, quimioterapia y apoyo para cáncer } \\
\text { de cérvix } \\
\text { Cáncer de mama } \\
\text { Colon y recto }\end{array}$ & $\begin{array}{l}2600 \\
3100 \\
5000\end{array}$ \\
\hline Cardiovasculares & $\begin{array}{l}\text { Paquete de cambio de comportamiento más tamizaje } \\
\text { de personas de alto riesgo }\end{array}$ & 150 & $\begin{array}{l}\text { Manejo clínico de hipertensión } \\
\text { Manejo clínico de hipercolesterolemia } \\
\text { Cuidado de angina estable } \\
\text { Cuidado de angina inestable o infarto } \\
\text { de miocardio } \\
\text { Angioplastía o bypass }\end{array}$ & $\begin{array}{r}2000 \\
4000 \\
100-200 \\
30000 \\
5000\end{array}$ \\
\hline
\end{tabular}

AVISA : año de vida saludable

IRA: infecciones respiratorias agudas

ETS: enfermedades de transmisión sexual

DN ID: diabetes no insulino dependiente

Fuente: referencia 26

nes preventivas de las ENT son al menos 20 veces más costo-efectivas que las clínicas en el ámbito de estas mismas enfermedades.

Todo ello indica que hay intervenciones preventivas de las ENT cuyo costo-efectividad compite con el de intervenciones para otros problemas. Aun en el caso de que no compitiese, no parece que la única alternativa sea esperar a que las ENT alcancen un estadio de desarrollo en el que sean necesarias las intervenciones clínicas que, sin lugar a dudas, son mucho menos costo-efectivas.

\section{Mito 6. No hay estrategias efectivas de prevención}

Finalmente, se duda de la efectividad de las intervenciones preventivas de ENT. Sin embargo, la evidencia científica existente demuestra que la prevención de 
los padecimientos no transmisibles funciona. Por ejemplo, la experiencia de los proyectos de Stanford, ${ }^{26}$ en Estados Unidos de América (EUA), y Karelia del Norte, en Finlandia, indica que el riesgo de enfermedad coronaria disminuye más de $20 \%$, tan sólo dos o tres años después de iniciada la intervención preventiva. En países menos desarrollados como la Isla de Mauricio ${ }^{27}$ o en el proyecto global de Cienfuegos, Cuba, ${ }^{28,29}$ también se han desarrollado intervenciones que han tenido impacto sobre diversos factores de riesgo.

Programas como el de Karelia del Norte han demostrado que se puede reducir la mortalidad de las enfermedades cardiovasculares mediante intervenciones dirigidas a disminuir la prevalencia de tan sólo tres factores de riesgo: tabaquismo, hipertensión e hipercolesterolemia. Gracias a esta estrategia, en Finlandia la mortalidad por enfermedad isquémica del corazón se ha reducido en $55 \%$ entre los hombres y en $68 \%$ entre las mujeres, desde 1972 hasta $1992 .{ }^{30}$ Hay que resaltar la importancia de las intervenciones sobre los factores de riesgo, ya que en este país la reducción de $13 \%$ en la prevalencia de hipercolesterolemia, de $9 \%$ en la de hipertensión arterial y de $70 \%$ en la de tabaquismo, ocurrida de 1972 a 1992 entre los hombres, predicen por sí solas una disminución de la mortalidad por enfermedad isquémica de 26,15 y $10 \%$, respectivamente. Entre las mujeres se aprecian tendencias similares, aunque las cifras de mortalidad son más bajas que entre los hombres.

Datos recientes de EUA abundan sobre la idea de la efectividad de las intervenciones en los factores de riesgo. Estos datos revelan que $50 \%$ de la disminución de la mortalidad por enfermedad isquémica del corazón ocurrida en la década de 1980 es atribuible a las intervenciones preventivas primarias y secundarias hechas en los tres importantes factores de riesgo mencionados (en un país con un modelo de intervención orientado principalmente al tratamiento clínico de estos problemas): tabaquismo, hipertensión e hipercolesterolemia. ${ }^{31}$

Ambos estudios indican además el efecto sinérgico que la intervención tiene cuando ésta es multifactorial -y no simplemente unifactorial- y cuando se integran estrategias poblacionales mediante intervenciones comunitarias en los determinantes de la salud, sin dejar de lado las intervenciones individuales, que son posibles gracias a los servicios clínicos preventivos.

\section{Conclusiones}

Definir qué enfermedades prevenir con los recursos disponibles es una cuestión fundamental de política de salud. A pesar de la evidencia que indica la magnitud y el impacto que la carga de las ENT tiene en América Latina y el Caribe, no son éstos los problemas que hasta ahora han tenido más prioridad. No hay datos sobre el gasto sanitario nacional dedicado a cada grupo de enfermedades, pero sí sobre los fondos de cooperación externa para todo el mundo, y son desoladores. Por cada unidad de carga de enfermedad atribuible a un problema transmisible, en 1990 se movilizaron 1.32 dólares estadunidenses, mientras que para cáncer se destinaron 0.05 centavos de dólar, y 0.01 para enfermedades cardiovasculares. ${ }^{32}$

Son muchos los motivos por los que se ha postergado la prevención de las ENT. En las páginas anteriores se han señalado los mitos que han relegado las intervenciones preventivas de estos problemas respecto a otros como los infecciosos o los materno-infantiles. El reto consiste en encontrar una política de salud equilibrada que resalte la importancia de las ENT sin olvidar la de otras enfermedades. No podría ser de otra manera si se quiere tener un verdadero impacto en la salud de las poblaciones. Por una parte, la distinción, a efectos de priorizar, entre la salud del adulto y la materno-infantil no tiene gran sentido, ya que la salud que tengan los niños determina en parte la que tendrán en la edad adulta y, a la vez, la salud de los adultos tiene un impacto inmediato en la de los niños.

Ejemplo de lo primero es que los adultos de talla baja, como consecuencia de retrasos en el crecimiento durante el periodo infantil, presentan una mayor mortalidad por enfermedades cardiovasculares o pulmonares obstructivas, ${ }^{33} \mathrm{o}$ bien, que los efectos del sobrepeso infantil se dejan sentir a lo largo de todo el ciclo de la vida. ${ }^{34}$ Ejemplo de lo segundo es que, en algunos países, la defunción de un adulto en la familia aumenta la probabilidad, hasta 12 veces, de una muerte entre los menores de su prole. ${ }^{35}$

Si llega a manifestarse el incremento proyectado de la carga que lleva consigo una ENT y a aumentar la demanda de servicios de salud por esta carga, los países de América Latina y el Caribe pueden encontrarse con serias limitaciones de recursos humanos y financieros que pueden poner en peligro las reformas del sector salud ahora en marcha. Además de la presión sobre el sistema de salud, se dejarán sentir las consecuencias económicas de la mortalidad y la discapacidad prematuras sobre la población económicamente activa. Si se adopta el enfoque que han seguido muchos países desarrollados para lidiar con las ENT, basado predominantemente en el manejo clínico de alta tecnología, existe el riesgo de que no haya recursos para atender ni siquiera las enfermedades transmisibles y materno-infantiles. 
Una política de salud adecuada y coherente con la realidad presente de América Latina y el Caribe no puede estar basada en la concepción de que hay una agenda inconclusa para la atención de las enfermedades transmisibles y materno-infantiles, y de que ésta debe cumplirse antes de abordar decididamente las ENT. La realidad es que la carga ocasionada por estas últimas actualmente es mayor que la de las demás enfermedades y tiende a aumentar, aunque coexista con otros problemas. De ahí la urgencia para iniciar programas e intervenciones de prevención primaria y secundaria de las ENT, con un costo relativamente bajo y un nivel de beneficios sustancioso, como el que representa el Programa CARMEN (Conjunto de Acciones para la Reducción Multifactorial de las Enfermedades no Transmisibles).*

\section{Agradecimientos}

Agradecemos al Dr. Fanklin White, cuyas ideas sirvieron de base para algunas de las secciones de este artículo.

\section{Referencias}

1. Bulatao RA. Mortality by cause. 1970 to 2015. En: Gribble JN , Preston S, ed. The epidemiological transition: Policy planning and implications for developing countries. W ashington, D.C.: N ational A cademy Press, 1993: 42-68.

2. Martin I. D ispelling myths about heart disease.W orld Health 1998;51(5): 6-7.

3. Sankaranarayanan R, Black RJ, Parkin D M. C ancer survival in developing countries. Lyon:International A gency for Research on C ancer/W orld Health O rganization (IARC Scientific Publication, núm. 145), 1998:135-157.

4. DCCT Research Group. The effect of intensive treatment of diabetes on the development and progression fo long-term complications insulindependent diabetes mellitus. N Engl J Med 1993;329:977-986.

5. UK Prospective Diabetes Study Group (UKPDS). Intensive blood-glucose control with sulphony lureas or insulin compared with conventional treatmente and risk complications in patients with type 2 diabetes. Lancet 1998;352:837-853.

6. W ilhelmsson C, Vedin JA, Elmfeldt D, Tibblin G, W ilhelmsen L. Smoking and myocardial infarction. Lancet 1975; I(7904):415-420.

7. Informe del C omité de Expertos de la 0 rganización Mundial de la Salud. Ginebra: O MS (Serie Informes Técnicos, núm. 568), 1974.

\footnotetext{
* Este programa actualmente está siendo implementado en ocho países de América Latina (Argentina, Brasil, Chile, Colombia, Costa Rica, Cuba, Puerto Rico y Uruguay) y en Canadá. Su finalidad es mejorar la salud de la población de un país, reduciendo la incidencia de los factores de riesgo asociados a las ENT mediante acciones combinadas y coordinadas de promoción de la salud, prevención de la enfermedad en el marco de la comunidad y de sus servicios de salud.
}

8. Fletcher CM, Peto R.The natural history of chronic airflow obstruction. BMJ 1977;1(6077):1645-1648.

9. Schell W D, Myers JN . Regression of atherosclerosis:A review. Prog C ardiovasc $D$ is 1997;39(5):483-496.

10. N icholls ES, Peruga A, Restrepo HE. Cardiovascular mortality in the Americas. World Health Stat Q 1993:46:134-150.

11. Hambrecht R, N iebauer J, Marburger C, G runze M, Kalberer B, Haver $\mathrm{K}$, et al.Various intensities of leisure time physical activity in patients with coronary artery disease: Effects on cardiorespiratory fitness and progression of coronary atherosclerotic lesions. J Am Coll Cardiol 1993;22:468477.

12. N iebauer J, Hambrecht R, Velich T, Haver K, M arburger C, Weiss $C$, et al. Attenuated progression of coronary artery disease after 6 years of multifactorial risk intervention: role of physical exercise. Circulation 1997; 96 (8):2534-2541.

13. W aters D. Lessons from coronary atherosclerosis "regression" trials. Cardiol Clin 1996;14:31-50.

14. Murray CJL, López AD. The global burden of diseases. Boston: Harvard University Press, 1996.

15. Martorell R, Kettel-Khan L, Hughes M, G rummer-Strawn. 0 besity in Latin A merican women and children.J N utr 1998;128:1464-1473.

16. D uncan BB, Rumel D, Zelmannowicz A, Serrate-Mengue S, D os Santos S, Dalmaz A. Social inequality in mortality in Sao Paulo State, Brazil. Int J Epidemiol 1995;24:359-365.

17. Corral F, Cueva P,Yépez J, Montes E. La baja escolaridad como factor de riesgo en el cáncer de cuello del útero. Bol 0 ficina Sanit Panam 1996; 121:511-517.

18.The IN CLEN Multicentre Collaborative Group. Socioeconomic status and risk factors for cardiovascular disease: A multicentre collaborative study in the international clinical epidemiology network (IN CLEN).J C lin Epidemiol 1994;47:1401-1409.

19. Duncan BB, Schmidt MI, A chutti AC, Polanczyk CA, Benia LR, Maia $A A G$. Socioeconomic distribution of noncommunicable disease risk factors in urban Brazil:The case of Porto Alegre. Bull Pan Am Health 0 rgan 1993;27:337-348.

20.0 rganización Pan A mericana de la Salud. Salud de las A méricas edición de 1998.W ashington DC: O PS, 1999.

21. Siegrist J. Social differentials in chronic diseases:W hat can sociological knowledge offer to explain and possibly reduce them? Soc Sci Med 1995; 41:1603-1605.

22. U nited States General Accounting 0 ffice. Infectious diseases: Soundness of W orld Health $O$ rganization estimates for eradication or elimination.W ashington, D.C., USG A0 : 1998:58.

23.W orld Health 0 rganization.T he glo bal world health report 1998. Ginebra:W HO, 1998.

24. Guldan GS. O bstacles to community health promotion. Soc Sci Med 1996:43:689-695.

25. Jamison DT. Disease control priorities in developing conuntries: $A n$ overview. En: Jamison DT, Mosley W H, Measham AR, Bo badilla JL, ed. D isease control priorities in developing conuntries. W ashington, D.C.: $0 \mathrm{X}$ ford Medical Publications, 1993.

26.W inkleby MA, Taylor CB, Jalutis D, Fortmann SP.T he long-term effects of a cardiovascular disease prevention trial:The Stanford Five-C ity project. Am J Public Health 1996;86:1773-1779.

27. Dowse GK, Gareebo H, Alberti KGMM, Zimmet $P$ et al. Changes in population cholesterol concentration and other cardiovascular risk factor levels after five years of the non-communicable disease intervention programme in Mauritius. BMJ 1995;311:1255-1259.

28. 0 rduñez PO, Espinosa AD,Alvarez O M, A polinaire J], Silva LC. Marcadores múltiples de riesgo para enfermedades crónicas no transmisibles: medición inicial del proyecto global de Cienfuegos, 1991-1992. La Habana: Instituto Superior de Ciencias Médicas, 1993.

29. Alvarez FC, Espinosa AD, Alvarez O M, Silva LC, Marrero RV. Marcadores de riesgo y enfermedades cardiovasculares en el Proyecto Global 
de Cienfuegos: estudio longitudinal 1992-1994. La Habana: Ministerio de Salud Pública, 1997.

30. Vartiainen $E$ et al. Do changes in risk factors in Finland explain the changes in ischemic heart disease mortality? En: Puska P et al.The N orth Karelia Project: 20 years results and experiences. Helsinky: $N$ ational Public Health Institute/H elsinky University Printing House, 1995.

31. Hunink $M G$ et al.The recent decline in mortality from coronary heart disease, 1980-1990. The effect of secular trends in risk factors and treatment. JAMA 1997;277(7):535-542.

32. Michaud $C$, Murray $C J L$. External assistance to the health sector in developing countries: A detailed analysis, 1972-1990. Bull World Health O rgan 1994;72:639-651.
33. W aaler HT. Height, weight and mortality. Acta Med Sandinavica 1984;S679;3-56.

34. Must A. Morbidity and mortality associated with elevated body weight in children and adolscentes. Am J C lin N utr 1996;63 suppl 1:445-S447.

35. $\mathrm{O}$ ver $\mathrm{M}$, Ellis RP, Huber JH, Solon 0. The consequences of adult illhealth. In the health of the adults in the developingo world. N ueva York: O xford University Press, 1994:161-199. 